\title{
Actes du Congrès Médico-Juridique du 18 novembre 2009
}

\author{
Musée Olympique, Lausanne, Suisse
}

\section{P. Rosatti}

L'histoire de l'assurance sociale est relativement récente dans sa conception actuelle et surtout son étendue, même si les structures d'aides, le plus souvent partielles, sont déjà anciennes. On peut évoquer l'Etablissement National des Invalides de la Marine (ENIM) qui existe toujours et remonte à l'époque de Louis XIV et Colbert en 1670. La Suisse connaît au début du XIX ${ }^{\mathrm{e}}$ siècle ses premières caisses de secours mutuels, puis plus tard dans le siècle, celles misent en place par l'industrie.

Par la suite, l'Europe s'est beaucoup inspirée de Bismarck, avec son modèle allemand des lois sur la Maladie (1883), l'Accident (1884) et l'Invalidité/vieillesse (1889).

Dans le courant du $\mathrm{XX}^{\mathrm{e}}$ siècle, chaque pays a développé ses propres systèmes de protection, avec certaines différences, mais aussi des ressemblances.

Pour les médecins, juristes, assureurs, il en est résulté au fil du temps une complexification de plus en plus grande du système, d'où parfois, si ce n'est souvent en raison des besoins de spécialisation, une communication de plus en plus rare entre les divers intervenants et acteurs de la santé, sans compter l'assuré qui est le plus souvent tout simplement perdu.

L'Organisation Mondiale de la Santé a donné sa définition de la santé, dans laquelle la dimension sociale est incluse, elle a aussi publié sa Classification Internationale des Maladies. Le lien entre santé et maladie n'est pas pour autant aisé à faire, que ce soit sur le plan médical ou juridique, la santé sociale n'est pas prise en compte, elle n'a pas valeur de maladie. La réponse à cette question n'est pas médicale, ni même juridique, elle serait plutôt du ressort du politique et ne peut pas être traitée ici, mais elle reste présente dans l'esprit de chacun et est souvent matière à conflit entre les intervenants.

Face à ce qui est pour beaucoup de médecins, juristes, services sociaux un nouveau paradigme médical, méconnu voire incompris, il importe à la fois de bien comprendre

Docteur Peter Rosatti $(\bowtie)$

CEMED SA, Avenue Perdtemps, 1260 Nyon (Suisse) les niveaux logiques de chacune des parties en cause, mais aussi d'être capable de transdisciplinarité ${ }^{1}$ et de communiquer avec chacun.

Le but de ce second congrès ${ }^{2}$ est ainsi la rencontre et l'échange entre les divers acteurs concernés sur des sujets parfois délicats, voire difficiles à comprendre ou même accepter.

Il sera abordé dans ces rencontres les questions d'arrêts de travail, des programmes qui favorisent la reprise, des diverses expériences en Europe sur ce sujet. La question de l'évaluation psychique sera discutée, domaine particulièrement difficile en raison de sa subjectivité potentielle. Les juristes discuteront encore de la difficile question de l'exigibilité du traitement et de la reprise de travail, ainsi que tout ce qui touche au secret médical et à la protection des données en assurances sociales.

Ce congrès est organisé par le Centre d'Expertise Médicale (CEMED) qui est une structure privée qui effectue des expertises mono ou pluridisciplinaires et intervient également dans diverses situations comme médecin conseil. Une trentaine de médecins et psychologues y sont associés, ils représentent pratiquement tous les domaines de la médecine.

Je tiens encore à remercier ici pour leur aide

- Les conférenciers pour la qualité de leurs textes ;

- Les coorganisateurs de ce congrès: Mme la Prof. B. Despland, les Drs J. Brinken et A Veya ;

- Les modérateurs : Mme la Prof. B. Despland et le Dr PA. Buchard ;

- SPRINGER France qui est l'éditeur de la revue Douleur et Analgésie et participe à cet événement en publiant les actes du congrès dans leur intégralité en plusieurs milliers d'exemplaires ;

- Mon secrétariat pour ce congrès : Mmes S. Roux et K. Rime

\footnotetext{
${ }^{1}$ La transdisciplinarité se réfère ici à la capacité de comprendre le modèle de pensée et de référence de l'autre.

${ }^{2}$ Le premier congrès en avril 2008 n'a pas été publié.
} 